ARTIGO ORIGINAL

\title{
Identidade de modelos para estimativa de biomassa aérea na Floresta Ombrófila Mista
}

\author{
Identity of models for aerial biomass estimation in Mixed Ombrophylous Forest
}

\author{
Vitor Hugo Zanette ${ }^{1}$ (D), Luciano Farinha Watzlawick ${ }^{1}$ (D), Richeliel Albert Rodrigues Silva ${ }^{1}$ (D), \\ Joelmir Augustinho Mazon ${ }^{1,2}$ (1) \\ ${ }^{1}$ Universidade Estadual do Centro-Oeste - UNICENTRO, Guarapuava, PR, Brasil \\ ${ }^{2}$ Centro Universitário UniGuairacá - UniGuairacá, Guarapuava-PR, Brasil
}

\begin{abstract}
Como citar: Zanette, V. H., Watzlawick, L. F., Silva, R. A. R., \& Mazon, J. A. (2021). Identidade de modelos para estimativa de biomassa aérea na Floresta Ombrófila Mista. Scientia Forestalis, 49(131), e3472. https://doi.org/10.18671/scifor.v49n131.09
\end{abstract}

\begin{abstract}
Resumo
O presente estudo teve como objetivo verificar se as seis regiões do Paraná podem ser representadas por uma única equação de biomassa, por meio do teste de identidade. Foram utilizadas as equações estabelecidas para áreas de Floresta Ombrófila Mista nos municípios de Araucária, Boa Ventura de São Roque, Castro, Coronel Vivida, General Carneiro e Reserva do Iguaçu. Para verificar a possível identidade entre os modelos foi aplicado o método de Regazzi, que consiste na comparação por meio do teste $F$, dos modelos completo e reduzido (hipótese $\mathrm{H}_{0}$ ). $\mathrm{O}$ teste $\mathrm{F}$ para as equações das seis áreas em estudo foi não significativo para $\alpha=5 \%$, para o modelo reduzido da equação selecionada $\left(F_{0}=0,311\right.$ e $\left.F_{\alpha}=1,69\right)$, aceitando-se $\mathrm{H}_{0}$. Portanto, o teste para a identidade de modelos mostrou-se adequado para analisar as diferentes características das regiões que são destacadas nas equações ajustadas. Além disso, o teste indicou com $95 \%$ de confiança que as seis regiões podem ser representadas por uma única equação.
\end{abstract}

Palavras-chave: Recursos florestais; Modelos de regressão; Equações alométricas.

\begin{abstract}
The present study aimed to verify if the six regions of Paraná can be represented by a single biomass equation by means of the identity test. The equations established for Araucaria Mixed Forest areas in the municipalities of Araucária, Boa Ventura de São Roque, Castro, Coronel Vivida, General Carneiro and Reserva do Iguaçu were used. To verify the possible identity between the models, the Regazzi method was applied, which consists in the comparison through the $\mathrm{F}$ test, the complete models and reduced (hypothesis $\mathrm{H}_{0}$ ). The $\mathrm{F}$ test for the equations of the six areas under study was not significant for $\mathrm{a}=5 \%$, for the reduced model of the selected equation $\left(F_{0}=0.311\right.$ and $\left.F_{\alpha}=1.69\right)$, accepting $H_{0}$. Therefore, the model identity test was adequate to analyze the different characteristics of the regions that are highlighted in the adjusted equations. In addition, the test indicated with 95\% confidence that the six regions can be represented by a single equation.
\end{abstract}

Keywords: Forest resources; Regression models; Allometric equations.

\section{INTRODUÇÃO}

A Floresta Ombrófila Mista (FOM), também conhecida como Floresta com Araucária, está inserida no domínio da Mata Atlântica, predominante no planalto meridional da região Sul do Brasil (Instituto Brasileiro de Geografia e Estatística, 1992). Atualmente, o estado do Paraná possui 1.866.412,42 ha de FOM, o que corresponde a 32,62\% da área original em fragmentos distribuídos em quase toda a extensão do estado (Accioly, 2013). A FOM abrange além das

Fonte de financiamento: Coordenação de Aperfeiçoamento de Pessoal de Nível Superior - Brasil (CAPES) - Código de Financiamento 001.

Conflito de interesse: Nada a declarar.

Autor correspondente: richelielufrn@gmail.com

Recebido: 10 setembro 2019.

Aceito: 17 dezembro 2020.

Editor: Paulo Henrique Müller Silva.

(c) (i) Este é um artigo publicado em acesso aberto (Open Access) sob a licença Creative Commons Attribution, que permite uso, distribuição e By reprodução em qualquer meio, sem restrições desde que o trabalho original seja corretamente citado. 
espécies endêmicas, muitas outras que formam comunidades interativas, com florística diferenciada, organização e estrutura ecológica (Sanquetta et al., 2005).

Como toda formação florestal inequiânea, a FOM também apresenta um elevado nível de complexidade e diversidade. Adicionalmente, essas possuem uma vasta gama de espécies, com várias alturas e tamanhos de copa, resultando em um perfil vertical irregular (Liboni et al., 2010). Devido a essas características, torna-se indispensável à utilização de técnicas de modelagem de crescimento e produção.

As informações obtidas na modelagem geram benefícios para o manejo sustentável das florestas. Entre as contribuições deste manejo destacam-se a conservação da floresta e de toda a sua biodiversidade, bem como benefícios socioeconômicos, recuperação de áreas degradadas, filtros biológicos para purificação da água ou a realização de projetos que se relacionem com o extrativismo de produtos não madeiráveis (Watzlawick et al., 2012).

Neste sentido, torna-se importante estudar continuamente a biomassa existente nos remanescentes florestais do estado do Paraná, principalmente na FOM. Uma das técnicas para estimar biomassa viva acima do solo em florestas tropicais, por meio de equações de regressão de uso global, foi desenvolvida por Brown et al. (1989).

Em estudos de análise de regressão, muitas vezes deseja-se saber se um conjunto de " $\mathrm{H}^{\prime \prime}$ equações ajustadas são idênticas e se o fenômeno pode ser representado por uma equação comum (Regazzi, 1993). A identidade de modelos permite exatamente isso, testar hipóteses relativas à identidade de modelos lineares e não-lineares. Os modelos lineares são utilizados pela facilidade em descrever o relacionamento aproximado, enquanto os modelos nãolineares tratam do relacionamento entre uma variável dependente e uma ou mais variáveis independentes, baseado em conhecimentos teóricos do problema tratado (Regazzi \& Silva, 2004).

$\mathrm{Na}$ área florestal, o teste de identidade de modelos é amplamente utilizado em povoamentos equiâneos (Andrade et al., 2015; Mendonça et al., 2015; Vendruscolo et al., 2015; Roveda et al., 2016; Araújo et al., 2018; Terra et al., 2018; Medeiros et al., 2018). No entanto, são incipientes estudos em remanescentes florestais da FOM, sendo observado apenas nos estudos realizados por Kurchaidt (2014) e Martins et al. (2017). Diante disso, o objetivo deste estudo foi aplicar o teste de identidade de modelos para verificar se as seis regiões do Paraná podem ser representadas por uma única equação de biomassa.

\section{MATERIAL E MÉTODOS}

\section{Áreas de estudo}

Foram utilizadas seis áreas amostrais implantadas em fragmentos de Floresta Ombrófila

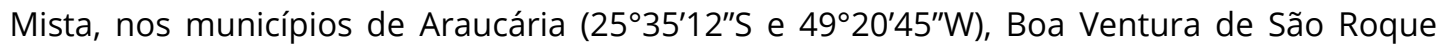

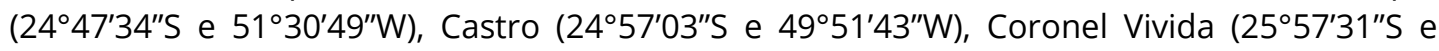

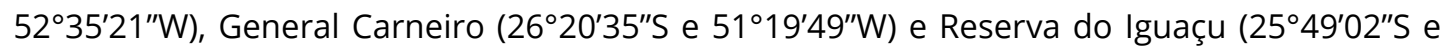
$\left.52^{\circ} 06^{\prime} 27^{\prime \prime} \mathrm{W}\right)$, no estado do Paraná. No total foram amostradas 382 árvores e anotados os valores do diâmetro a altura do peito e da biomassa aérea seca. Os fragmentos florestais onde estão instaladas as áreas experimentais possuem variadas topografias, solos e níveis de antropização, mas todas encontram-se em estágio secundário de sucessão, classificadas conforme a Resolução n² 2 - CONAMA/1994 (Brasil, 1994).

O fragmento localizado no município de Araucária é constituído de FOM Aluvial, com 897 m; os demais fragmentos são de FOM Montana e Altomontana, com altitudes entre 630 e $1.023 \mathrm{~m}$. O clima das áreas dos fragmentos, de acordo com a classificação de Köppen é Subtropical Úmido Mesotérmico, com verões frescos, sem estações secas e com geadas (Cfb) (Alvares et al., 2013). 


\section{Análise dos dados}

Frente ao objetivo de ajustar um modelo de regressão com a junção das seis áreas, definiu-se um conjunto de todas as observações, denominando-o de área Paraná. Ao todo foram amostradas 382 árvores, sendo 102 em Araucária, 26 em Boa Ventura de São Roque, 42 em Castro, 17 em Coronel Vivida, 172 em General Carneiro e 23 em Reserva do Iguaçu.

Com a definição dessa área, avaliou-se qual modelo melhor representa a junção das seis áreas individuais. Por fim, utilizou-se o teste de identidade de modelos para verificar se as seis regiões podem ser representadas por uma única equação. Dentre os modelos ajustados para cada área, aquele que obteve as melhores estatísticas para a maioria das áreas em estudo foi submetido ao teste de identidade de modelos proposto por Regazzi (1993), a fim de verificar se as estimativas dos parâmetros para os modelos completo e reduzido são estatisticamente iguais.

Para isso, foi realizado o teste $F$ para testar duas hipóteses: a nula $\left(\mathrm{H}_{0}\right)$, quando o modelo completo é estatisticamente igual ao modelo reduzido; e a alternativa $\left(\mathrm{H}_{1}\right)$, quando o modelo completo não é estatisticamente igual ao modelo reduzido. As etapas para efetuar os cálculos do teste de identidade estão apresentadas na Tabela 1.

Tabela 1: Análise de variância para o teste de identidade do modelo de biomassa em seis áreas do estado do Paraná.

\begin{tabular}{ccccc}
\hline Fonte de variação & $\mathbf{G L}$ & SQ & QM & $\boldsymbol{F}_{\mathbf{O}}$ \\
\hline Parâmetros $(\beta)$ & $(H \cdot p)$ & $Q_{1}=\hat{\beta}^{\prime} X^{\prime} Y$ & & \\
Parâmetros $(\mathrm{a})$ & $p$ & $Q_{2}=\hat{\theta}^{\prime} Z^{\prime} Y$ & \\
Redução $\left(\mathrm{H}_{0}\right)$ & $(H-1) \cdot p$ & $Q_{3}=Q_{1}-Q_{2}$ & $V_{1}=\frac{Q_{3}}{(H-1) \cdot p}$ & $\frac{V_{1}}{V_{2}}$ \\
Resíduo & $(N-H \cdot p)$ & $Q_{4}=Q_{5}-Q_{1}$ & $V_{2}=\frac{Q_{4}}{(N-H \cdot p)}$ \\
Total & $N$ & $Q_{5}=Y^{\prime} Y$ & \\
\hline
\end{tabular}

$\mathrm{GL}$ = grau de liberdade; $\mathrm{SQ}=$ soma dos quadrados; $\mathrm{QM}=$ quadrado médio; $\mathrm{F}=$ teste $\mathrm{F}$.

\section{RESULTADOS E DISCUSSÃO}

Foi testada por meio de identidade de modelos a possiblidade de indicar uma equação para estimar a biomassa arbórea no estado do Paraná. Após análise e escolha das equações que representam cada uma das seis áreas estudadas, o modelo de equação $B=a+b \cdot(D A P)+c \cdot(D A P)^{2}$ apresentou os melhores resultados estatísticos para cada área, conforme Zanette et al. (2017).

As equações ajustadas para os diferentes remanescentes apresentaram coeficientes de determinação ajustado ( $R^{2}$ aj.) de 0,23 a 0,93 e erro padrão médio (Syx\%) entre 34,4\% e 85,83\%. $A$ verificação se as áreas poderiam ser representadas por uma única equação utilizando a metodologia empregada por Regazzi (1993), que consiste na comparação por meio do teste $\mathrm{F}$, dos modelos completo e reduzido (hipótese $\mathrm{H}_{0}$ ) está descrita na Tabela 2.

Tabela 2. Análise de variância relativa ao teste de hipótese $\mathrm{H}_{0}$ entre as equações de biomassa arbórea em seis áreas do estado do Paraná.

\begin{tabular}{ccccc}
\hline Fonte de variação & GL & SQ & QM & $\boldsymbol{F}_{\boldsymbol{O}}$ \\
\hline Parâmetros $(\beta)$ & 18 & 123.075 .181 & & \\
Parâmetros $(\alpha)$ & 3 & $122.979 .147,2$ & & \\
Redução $\left(\mathrm{H}_{0}\right)$ & 15 & $96.033,7$ & $6.402,2$ & $0,311^{\text {ns }}$ \\
Resíduo & 364 & $7.490 .932,3$ & $20.579,5$ & \\
Total & 382 & $130.566 .113,3$ & & \\
\hline
\end{tabular}

GL = grau de liberdade; SQ = soma dos quadrados; $\mathrm{QM}=$ quadrado médio; F = teste F. ns: não significativo ao nível de $5 \%$ de probabilidade. 
A estatística $\mathrm{F}$ mostra o quanto o modelo ajustado representa na relação entre a variável dependente e as variáveis independentes, sendo preferíveis modelos com estatística $\mathrm{F}$ elevada (Schneider, 1997). Portanto, nota-se que no teste $F$, as equações das seis áreas em estudo foram não significativas, considerando $\alpha=5 \%$, para o modelo reduzido da equação selecionada ( $F_{0}=0,311$ e $\left.F_{a}=1,69\right)$, aceitando-se $H_{0}$. Diante disso, a não existência de diferença significativa entre os modelos completo e reduzido leva a concluir que o ajuste da equação $B=79,443-12,130 \cdot D A P+0,655 \cdot D A P^{2} \quad$ (Zanette et al., 2017) foi adequado para quantificar a biomassa das seis áreas com Floresta Ombrófila Mista, no estado do Paraná, com 95\% de confiança. Porém, nem sempre é possível obter bons ajustes em povoamento com grande amplitude diamétrica, sendo indicados modelos estratificados em classes de diâmetro, como foi observado na estimativa de volume em povoamento de Araucaria angustifolia, no município de Campo Largo, PR (Curto et al., 2014).

Regazzi \& Silva (2004) utilizaram o teste da razão de verossimilhança com aproximações pelas estatísticas do qui-quadrado e $\mathrm{F}$ para testar a hipótese de igualdade de subconjunto de parâmetros e, a identidade de modelos de regressão não-linear para dados com repetições de experimentos com delineamento inteiramente casualizado. Assim, eles concluíram que em amostras suficientemente grandes $(N>120)$, as duas aproximações são praticamente equivalentes. Neste sentido, pode-se afirmar que os resultados obtidos neste estudo, por meio do teste $F$, foram representativos para estimar a biomassa arbórea.

Ao estudar diferentes situações experimentais admitindo um modelo para cada situação, verifica-se que os modelos são idênticos, mostrando possível ou não a representação do conjunto de equações por meio de uma equação comum. Com isso, em trabalho com identidade de modelos para a curva do coletor (curva espécie-área), Kurchaidt (2014) avaliando quatro parcelas permanentes de inventário florestal no município de São João do Triunfo/PR, denominadas Araucária, Rio, Imbuia e Fogo, em quatro anos distintos (1995/2000/2005/2010), não conseguiu representar as quatro equações apenas por uma. Diferentemente do que foi realizado no presente estudo, que registrou a biomassa apenas em um dado momento, a dinâmica das espécies arbóreas, provavelmente, interferiu na identidade de modelos.

Em outro estudo, Martins et al. (2015) aplicando o teste de identidade de modelos volumétricos para povoamentos de Eucalyptus sp. com idades variando entre 5 e 6,5 anos, plantados em diferentes espaçamentos, em sete municípios de Minas Gerais, não obtiveram uma equação comum para todos os municípios, mesmo as equações apresentando formas semelhantes em níveis diferentes, ao nível de 5\% de significância. Vendruscolo et al. (2015) avaliando a identidade de modelos, em diferentes espaçamentos de Tectona grandis, no município de Cáceres, MT, agruparam a maioria dos espaçamentos em uma única equação, otimizando, consequentemente, o tempo e os custos no manejo da espécie.

Assim, observa-se que apesar dos remanescentes florestais avaliados no presente estudo serem inequiâneos, o ajuste foi mais preciso do que os realizados nos povoamentos naturais ou plantados citados anteriormente. Provavelmente, isso ocorre devido às características morfológicas similares entre as espécies arbóreas das áreas analisadas.

Dessa forma, recomenda-se que as amostras de árvores para avaliação de biomassa contemplem as diversas espécies de uma dada região, preferencialmente com árvores de diâmetros maiores que $30 \mathrm{~cm}$, pois o número de informações de árvores pequenas disponíveis é suficiente para bons ajustes, como também que o número de indivíduos de cada unidade seja representativo, permitindo assim melhores estimativas de biomassa. Além da estratificação por espécie, indica-se também por grupo ecológico, tornando o ajuste ainda mais eficiente, conforme observado por Baptista et al. (2019), em fragmento de Mata Atlântica, no município de Seropédica, RJ.

Curto et al. (2018) afirmaram que para a diminuição de custos e tempo no desbaste em floresta natural, indica-se o aumento da precisão e estratificação, visando a redução da intensidade amostral. Os resultados obtidos poderão integrar planos de manejo e derrubadas 
de árvores das regiões estudadas, como confirmada esta possibilidade em povoamento natural de Eremanthus incanus, no município de Morro do Pilar, MG (Ferreira et al., 2014).

Além disso, as informações geradas permitirão alimentar os bancos de dados em áreas que compreendem, por exemplo, PCHs (Pequenas Centrais Hidrelétricas), bem como no fornecimento de uma equação que represente a estimativa de biomassa aérea para o estado do Paraná.

\section{CONCLUSÕES}

$O$ teste para a identidade de modelos mostra-se adequado para analisar as diferentes características das regiões com Floresta Ombrófila Mista do estado do Paraná. O teste também indica, com $95 \%$ de confiança, que as seis regiões podem ser representadas por uma única equação.

\section{REFERÊNCIAS}

Accioly, P. (2013). Mapeamento dos remanescentes vegetais arbóreos do estado do Paraná e elaboração de um sistema de informações geográficas para fins de análise ambiental do estado (Tese de doutorado). Universidade Federal do Paraná, Curitiba.

Alvares, C. A., Stape, J. L., Sentelhas, P. C., Gonçalves, J. L. M., \& Sparovek, G. (2013). Köppen's climate classification map for Brazil. Meteorologische Zeitschrift, 22(6), 711-728.

Araújo, E. J. G., Loureiro, G. H., Sanquetta, C. R., Sanquetta, M. N. I., Corte, A. P. D., Péllico Netto, S., \& Behling, A. (2018). Allometric models to biomass in restoration areas in the Atlantic rain forest. Floresta e Ambiente, 25(1), 1-13.

Andrade, V. C. L., Kroetz, E. A., Nicola, A., Souza, P. B., Nohama, F. K., Leite, H. G., Binoti, D. H. B., \& Binotil, M. L. M. S. (2015). Amostragem e agrupamento de dados de relação hipsométrica em inventários florestais de Cerrado Tocantinense. Pesquisa Florestal Brasileira, 35(83), 227-238.

Baptista, F. R. C. B., Ataíde, D. H. S., Rocha, P. V., Silva, L. C., Araújo, E. J. G., Curto, R. A., \& Mendonça, B. A. F. (2019). Estratificação no ajuste de modelos de relação hipsométrica em fragmento de floresta inequiânea. Scientific Electronic Archives, 12(6), 52-61.

Brasil. Resolução ${ }^{\circ} 2$, de 18 de março de 1994. Define formações vegetais primarias e estágios sucessionais de vegetação secundária, com finalidade de orientar os procedimentos de licenciamento de exploração da vegetação nativa do Paraná. Diário Oficial [da] República Federativa do Brasil, Brasília, n. 059, 28 mar. 1994.

Brown, S., Gillespie, A. J. R., \& Lugo, A. E. (1989). Biomass estimation methods for tropical forests with applications to forest inventory data. Forest Science, 35, 881-902.

Curto, R. A., Wink, C., Araújo, E. J. G., \& Kohler, S. V. (2018). Modelos de relação hipsométrica por classe de aproveitamento em floresta de transição Cerrado-Amazônia no Mato Grosso. Enciclopédia Biosfera, 15(28), 687-700.

Curto, R. D. A., De Mattos, P. P., Braz, E. M., Zachow, R., \& Péllico Netto, S. (2014). Caracterização da estrutura e desenvolvimento de modelos para quantificação do potencial madeireiro em povoamento não manejado de Araucaria angustifolia. Pesquisa Florestal Brasileira, 34(80), 303-319.

Ferreira, G. W. D., Ferraz Filho, A. C., Pinto, A. L. R., \& Scolforo, J. R. S. (2014). Influência do desbaste na forma do fuste de povoamentos naturais de Eremanthus incanus (Less.) Less. Semina, 35(4), 17071720.

Instituto Brasileiro de Geografia e Estatística - IBGE. (1992). Manual técnico da vegetação brasileira. Rio de Janeiro: IBGE.

Kurchaidt, S. M. (2014). Uso de métodos estatísticos multivariados em dinâmica florestal (Tese de doutorado). Universidade Federal do Paraná, Curitiba.

Liboni, A. P., Rodrigues, D. R., Perina, B. B., Rosa, V. P. P., Bovolenta, Y. R., Bianchini, E., \& Pimenta, J. A. (2010). Relações alométricas da comunidade arbórea de diferentes áreas de uma Floresta Ombrófila Mista do Sul do Brasil. Semina, 31(2), 125-136.

Martins, A. P. M., Debastiani, A. B., Pelissari, A. L., Machado, S. A., \& Sanquetta, C. R. (2017). Estimativa do afilamento do fuste de araucária utilizando técnicas de inteligência artificial. Floresta e Ambiente, $24,1-10$. 
Martins, R. M., Leite, M. V. S., Cabacinha, C. D., \& Assis, A. L. (2015). Teste de identidade de modelos volumétricos para povoamentos de Eucalyptus sp. em sete municípios de Minas Gerais. Enciclopédia Biosfera, 11(21), 1818-1833.

Medeiros, R. A., Paiva, H. N., D'Ávila, F. S., \& Leite, H. G. (2018). Growth and yield of teak stands at different spacing. Pesquisa Agropecuária Brasileira, 53(10), 1109-1118.

Mendonça, A. R., Pacheco, G. R., Vieira, G. C., Araújo, M. S., \& Interamnense, M. T. (2015). Identidade de modelos para estimativa do volume de pinus. Nativa, 3(4), 281-286.

Regazzi, A. J. (1993). Teste para verificar a identidade de modelos de regressão e a igualdade de alguns parâmetros num modelo polinomial ortogonal. Revista Ceres, 40(228), 176-195.

Regazzi, A. J., \& Silva, C. H. O. (2004). Teste para verificar a igualdade de parâmetros e a Identidade de modelos de regressão não-linear. I. Dados no delineamento inteiramente casualizado. Revista de Matemática e Estatística, 22(3), 33-45.

Roveda, M., Dias, A. N., Figueiredo Filho, A., Scavinski, V., \& Castro, R. R. (2016). Testes de identidade de modelos aplicados na construção de curvas de índices de local e na produção de povoamentos de Eucalyptus grandis. Scientia Forestalis, 44(109), 115-127.

Sanquetta, C. R., Dalla Corte, A. P., Vulcanis, L., \& Berni, D. M. (2005). Sobrevivência de mudas de Araucaria angustifolia perante o controle de taquaras (Bambusoideae) no Paraná, Brasil. Revista Floresta, 35(1), 127-135.

Schneider, P. R. (1997). Análise de regressão aplicada à engenharia florestal. Santa Maria: UFSM.

Terra, D. L. C. V., Andrade, V. C. L., \& Freitas, B. C. (2018). Identidade de modelos volumétrico e de afilamento para Corymbia citriodora. Advances in Forestry Science, 5(1), $269-274$.

Vendruscolo, D. G. S., Chaves, A. G. S., Silva, R. S., Souza, H. S., Medeiros, R. A., Motta, A. S., \& Silva, F. T. (2015). Identidade em modelos hipsométricos para Tectona grandis com diferentes espaçamentos em Cáceres-MT. Nativa, 3(1), 44-49.

Watzlawick, L. F., Caldeira, M. V. W., Viera, M., Schumacher, M. V., Godinho, T. O., \& Balbinot, R. (2012). Estoque de biomassa e carbono na Floresta Ombrófila Mista Montana Paraná. Scientia Forestalis, 40(95), 353-362.

Zanette, V. H., Kurchaidt, S. M., Camargo, L. P., Watzlawick, L. F., \& Koehler, H. S. (2017). Ajuste de modelos de regressão para a estimativa da biomassa aérea para seis regiões do estado do Paraná. Enciclopédia Biosfera, 14(26), 29-43.

Contribuição dos Autores: VHZ: conceituação, curadoria de dados, análise formal, investigação, metodologia, validação, visualização, escrita - primeira redação, escrita - revisão e edição; LFW: conceituação, curadoria de dados, investigação, metodologia, administração do projeto, recursos, supervisão, escrita - primeira redação; RARS: conceituação, investigação, metodologia, supervisão, escrita - primeira redação, escrita - revisão e edição; JAM: conceituação, metodologia, supervisão, escrita - primeira redação. 